\title{
ANATOMIA DOS LENHOS DE MIMOSA INTRICATA BENTH. E MIMOSA TAIMBENSIS BURKART (FABACEAE) ${ }^{1}$
}

\author{
PAULO FERNANDO DOS SANTOS MACHADO² JOSÉ NEWTON CARDOSO MARCHIORI ${ }^{3}$
}

\section{RESUMO}

Os lenhos de Mimosa intricata e Mimosa taimbensis apresentam características anatômicas comuns em Leguminosae, salientando-se: placas de perfuração simples, pontoações intervasculares ornamentadas e em arranjo alterno, parênquima paratraqueal e fibras libriformes. Em Mimosa intricata predominam poros em múltiplos racemiformes, caráter aparentemente restrito a espécies da série Stipellares Benth. Mimosa taimbensis, por sua vez, separa-se, facilmente, pelos poros solitários e em pequenos múltiplos, bem como pela estratificação parcial de parênquima axial e elementos vasculares.

Palavras-chave: anatomia da madeira, Caesalpinioideae, Calothamnos, Fabaceae, Mimosa intricata, Mimosa taimbensis, Mimosoideae, Stipellares.

\section{ABSTRACT}

[Wood anatomy of Mimosa intricata Benth. and Mimosa taimbensis Burkart (Fabaceae)].

Mimosa intricata and Mimosa taimbensis present some anatomical features that are common among Leguminosae woods: simple perforation plates, alternate vestured pits, paratracheal parenchyma and libriform fibers. Mimosa intricata shows vessels in large clusters, being this character apparently restricted, among Mimosa species, to those of Stipellares series. Mimosa taimbensis, on the other hand, can be distinguished by having solitary and small multiple pores, as well as storied structure of axial parenchyma and vessel elements. Key words: Caesalpinioideae, Calothamnus, Fabaceae, Mimosa intricata, Mimosa taimbensis, Mimosoideae, Stipellares, wood anatomy.

\section{INTRODUÇÃO}

Pertencente à família Fabaceae e subfamília Caesalpinioideae (LPWG, 2017), o gênero $M i$ mosa L. compreende cerca de 530 binômios válidos e em sua grande maioria da região Neotropical, apresentando 31 espécies na ilha de Madagascar e um número ainda menor no leste da África e sudeste da Ásia (Coutinho, 2009). Devido à diversificação recente dos clados, que emergiram, em sua maioria, entre 15 e 3 milhões de anos a.P., essa distribuição geográfica se explica pela teoria da dispersão a longa distância e descarta, segundo Simon

1 Recebido em 13-09-2016 e aceito para publicação em 18-02-2017.

2 Engenheiro Florestal, doutorando do Programa de Pós-Graduação em Engenharia Florestal, Universidade Federal de Santa Maria. Santa Maria, RS, Brasil. barcasole@gmail.com

3 Engenheiro Florestal, Dr. Bolsista de Produtividade em Pesquisa (CNPq - Brasil). Professor Titular do Departamento de Ciências Florestais, Universidade Federal de Santa Maria. marchiori@pq.cnpq.br
(2008), qualquer hipótese de vicariância pela separação dos continentes.

Na taxonomia do gênero Mimosa L. se destacam dois estudos magistrais, distanciados entre si pelo lapso de mais de cem anos.

Em meados do século dezenove, Bentham (1842) colocou as espécies de Mimosa em duas seções - Eumimosa DC. e Habbasia Benth. -, distintas entre si pela presença de flores haplostêmones e diplostêmones, respectivamente. Em sua monografia para a Flora Brasiliensis, Bentham (1876) reconheceu nove séries botânicas na seção Habbasia, salientando-se Stipellares Benth., segregada das demais com base na presença de capítulos globosos, legumes subplanos de valvas indivisas, estipelas orbiculares ou ovais e folíolos pouco abundantes, entre outros caracteres morfológicos. Para a seção Eumimosa DC., Bentham (1876) reconheceu doze séries botânicas, entre as quais Lepidotae, distinta das demais por reunir plantas inermes e com pinas uni ou plurijugas, 
revestidas por tomento estrelado-plumoso ou lepidoto.

Em Sensitivae Censitae, Barneby (1991) elaborou um novo (e complexo) esquema taxonômico para Mimosa L., composto de cinco seções (Batocaulon DC., Calothamnos Barneby, Habbasia DC., Mimadenia Barneby e Mimosa L.), 41 séries e 37 subséries. A partir de ancestrais Piptadenioides, a seção Mimadenia foi a primeira a ser tratada em seu "hipotético" esquema filogenético, seguida pela seção Batocaulon, a qual teria dado origem às seções Calothamnos e Habbasia; a partir desta última, por fim, ter-se-ia derivado a seção Mimosa. De acordo com Barneby (1991), a série Stipellares Benth. foi agregada a outras 24 para compor a seção Batocaulon DC., que se distingue, do conjunto, pela presença de flores tetrâmeras e em capítulos (com exceção de Mimosa barnebiana, que possui espigas verdadeiras), de estípulas em forma variável, embora nunca foliáceas, e de parafilídios elípticos, ovados, reniformes ou suborbiculares, simulando diminutos folíolos. A série Lepidotae Benth., por sua vez, foi elevada por Barneby (1991) ao status de seção, sob o nome de Calothamnos.

A respeito da classificação infragenérica de Mimosa L., estudos filogenéticos recentes (Luckow et al., 2000; Sulaiman et al., 2003; Bessega et al., 2008; Simon et al., 2011) demonstram que o gênero compõe um grupo monofilético, embora não se possa dizer o mesmo da maioria das seções e séries propostas por Rupert Charles Barneby (Santos-Silva et al., 2015).

O presente estudo visa à descrição microscópica dos lenhos de Mimosa intricata Benth. e Mimosa taimbensis Burkart, que, até o momento, seguem anatomicamente desconhecidos.

Arbusto baixo e com ramos espinhosos densamente emaranhados (Rambo, 1966), Mimosa intricata vincula-se à série Stipellares Benth. Trata-se de espécie endêmica de campos pedregosos do Rio Grande do Sul, onde cresce em meio à vegetação arbustiva (Rambo, 1966). Com
Mimosa bifurca Benth., a espécie compartilha a presença de glândulas impressas no dorso dos folíolos, diferindo, todavia, entre outros caracteres morfológicos, por ser planta armada de acúleos (vs. inerme em $M$. bifurca) e com pecíolos mais curtos (8-18mm vs. 20-70mm). Embora nativa na Serra do Sudeste do Rio Grande do Sul, cuja geologia prolonga-se no interior do Uruguai, Mimosa intricata não ocorre nesse país, de acordo com Izaguirre \& Beyhaut (2003).

Nativa na "região serrana" dos estados de "Santa Catarina e Rio Grande do Sul" (Burkart, 1964), Mimosa taimbensis Burkart é espécie "exclusiva" da "matinha nebular" dos "aparados da Serra Geral", no "alto dos taimbés", ocorrendo, por vezes, como indivíduos "isolados" na "borda oriental dos campos" (Klein, 1979). Sob o ponto de vista taxonômico, a espécie insere-se ou na série Lepidotae (sensu Bentham, 1876), ou na seção Calothamnos (Barneby, 1991).

Além das descrições anatômicas, o presente trabalho visa a realizar uma análise taxonômica dos lenhos com vistas ao reconhecimento de caracteres de valor diagnóstico para os táxons a que pertencem ambas as espécies, valendo-se, para isso, de dados anatômicos relativos a outros binômios, colhidos da literatura.

\section{REVISÃO DE LITERATURA}

Apesar do escasso interesse econômico da madeira na maioria das espécies de Mimosa L., a literatura anatômica já dispõe de descrições microscópicas de numerosos binômios, justificando a busca de vinculações anátomotaxonômicas.

Para o conjunto das Leguminosas (Fabaceae), Senn (1943) e Record \& Hess (1949) consideraram vantajosa a manutenção de sua unidade, devido à inexistência de uma nítida diferenciação anatômica entre as subfamílias. Metcalfe \& Chalk (1972), ao contrário, trataram Mimosaceae, Caesalpiniaceae e Papilionaceae como distintas, reconhecendo Mimosaceae como a família anatomicamente menos 
evoluída e Papilionaceae como a mais especializada.

Em estudo sobre a madeira de Leguminosas argentinas, Cozzo (1951) considerou que a estrutura mais homogênea de Mimosoideae e Caesalpinioideae, em relação às Papilionoideae, dificulta o reconhecimento de diferenças anatômicas entre suas tribos e gêneros. No caso de Mimosoideae, o autor reconheceu a existência de variação anatômica ainda menor do que em Caesalpinioideae.

Metcalfe \& Chalk (1972) consideraram os raios lenhosos de Mimosaceae como mais especializados, comparado às outras duas famílias, por serem invariavelmente homogêneos, de células procumbentes e com unisseriados relativamente escassos. Os autores reforçaram suas conclusões filogenéticas afirmando que, apesar do elevado e uniforme desenvolvimento radial das Mimosaceae, em Caesalpiniaceae e, sobretudo, em Papilionaceae se encontram tanto raios ainda mais especializados como tipos menos evoluídos.

Baretta-Kuipers (1981), em estudo com 35 gêneros de Mimosoideae, 85 de Caesalpinioideae e 68 de Papilionoideae, comprovou que a estrutura dos raios é o caráter anatômico mais importante na taxonomia das Leguminosas, e que Caesalpinioideae apresenta a estrutura menos evoluída da família. Segundo a autora, esta subfamília constitui a base, a partir da qual a especialização anatômica seguiu dois rumos distintos: em direção à Mimosoideae, com a especialização dos raios, e em direção a Papilionoideae, pela tendência à estratificação de todos os elementos. É interessante notar que Burkart (1952) chegou, praticamente, à mesma conclusão, com base em caracteres da morfologia externa.

Em estudo anatômico do lenho de alguns gêneros sulamericanos, Reinders-Gouwentak (1955) observou que o caráter estrutura estratificada permite dividir as Leguminosae em duas subfamílias: Mimosaceae e Papilionaceae, a última das quais incluindo Caesalpinioideae.
Para a identificação de tribos de Leguminosae, Baretta-Kuipers (1981) ressalta, como importantes, o tipo de raios (homocelulares ou heterocelulares), a largura dos mesmos (uni ou multisseriados) e a ocorrência de estratificação. Para a autora, o arranjo do parênquima axial também se mostra importante para a segregação de gêneros e espécies.

No caso de Mimosa L., Baretta-Kuipers (1981) destaca que o lenho pode ser facilmente reconhecido pela ausência de estratificação completa e pelos raios sempre homocelulares, com células procumbentes e de seção pequena, em plano tangencial, baixas em plano radial. A autora ressalta que esse último aspecto raramente se encontra em Caesalpinioideae, estando, neste caso, associado à estratificação de raios.

Em seu estudo sobre espécies argentinas de Leguminosas Mimosoídeas e Cesalpinioídeas, Cozzo (1951) referiu-se a Mimosa L. como táxon "estruturalmente heterogêneo", por não ter reconhecido a existência de um traço anatômico relevante, comum às espécies investigadas. De fato: apesar de ainda pouco estudado, face ao elevado número de suas espécies, a diversidade estrutural atribuida ao gênero compara-se ao descrito para o conjunto das Mimosoideae.

Um dos primeiros estudos sobre o lenho em Mimosa L. deve-se a Record \& Hess (1949), autores que, entretanto, apenas mencionam aspectos gerais e organolépticos, com base em $M$. bracaatinga Hoehne (atual M. scabrella Benth.) e em M. schomburgkii Benth., espécie da Guiana. Cabe destacar, ainda, que Metcalfe \& Chalk (1972) também não mencionaram o gênero em sua diagnose anatômica das Mimosaceae.

Em chave anatômica para 13 espécies de Mimosa nativas na Argentina, Cozzo (1951) destacou os seguintes caracteres:

- Anéis de crescimento fracamente definidos, melhor demarcados em Mimosa ostenii.

- Porosidade difusa, com tendência a circular em Mimosa ostenii; em arranjo dendrítico 
"bem notável" em $M$. hassleriana e $M$. uliginosa; e apenas tendente a esse arranjo em M. cruenta e $M$. uruguensis.

- Vasos solitários e em número de 2-3, menos comumente 4-5 poros; solitários e em múltiplos de 2, em Mimosa scabrella e M. adpressa; compondo longos múltiplos, nas espécies com porosidade dendrítica. Vasos de seção oval, angulares e de paredes delgadas em Mimosa pigra, espessas em $M$. polycarpa.

- Elementos vasculares retilíneos ou levemente sinuosos, com placas de perfuração simples, oblíquas; apêndices ausentes ou curtos, por vezes longos.

- Pontoações intervasculares ornamentadas, alternas, pequenas a médias; grandes, em $\mathrm{Mi}$ mosa pigra. Pontoações raio-vasculares e parênquimo-vasculares, semelhantes às intervasculares; semiareoladas, em $M$. pigra.

- Tilos e espessamentos espiralados, geralmente ausentes; espessamentos delgados, em elementos muito curtos de Mimosa cruenta.

- Traqueóides vasculares, escassos, em espécies de porosidade dendrítica.

- Raios heterogêneos até quase homogêneos; exclusivamente unisseriados, em Mimosa pigra; predominantemente unisseriados, em $M$. uruguensis; geralmente trisseriados, em $M$. adpressa; e com multisseriados estreitos (até cinco células), em $M$. bimucronata e $M$. polycarpa. Raios com células envolventes curtas, em poucas espécies; com células radiais oleosas, em M. bimucronata.

- Parênquima axial abundante ou muito abundante em Mimosa cruenta, M. detinens e $M$. hassleriana, menos desenvolvido em $M$. adpressa, M. bimucronata e M. scabrella; no primeiro grupo, do tipo paratraqueal-em-faixas, comumente curto-confluente. Células parenquimáticas axiais fusiformes e em séries, com predomínio de fusiformes em $M$. pigra.

- Fibras libriformes muito curtas, de paredes finas a semiespessas, frequentemente septadas; fibrotraqueóides, presentes em Mimosa polycarpa.
- Estratificação, incompleta, em Mimosa adpressa e M. scabrella.

Das espécies de Mimosa nativas no Rio Grande do Sul, a mais investigada no tocante à anatomia do lenho é Mimosa scabrella Benth., mercê de seu interesse florestal e madeireiro (Tortorelli, 1956; Richter \& Charvet, 1973; Marchiori, 1995; Marchiori et al., 2009). Da mesma seção Calothamnos Barneby, dispõe-se, ainda, de descrições anatômicas de Mimosa berroi Burkart (Marchiori, 1996a), Mimosa daleoides Benth. (Marchiori 1982; Marchiori et al., 2011), Mimosa eriocarpa Benth. (Carnieletto \& Marchiori, 1993; Marchiori et al., 2011), Mimosa incana (Marchiori, 1996b; Marchiori et al., 2010), Mimosa flocculosa Burkart (Pereira et al., 2013) e Mimosa pilulifera Benth. (Marchiori \& Muñiz, 1997a; Marchiori et al., 2010).

Com relação à série Stipellares Benth., foram anatomicamente descritos os lenhos de Mimosa barnebiana e Mimosa bifurca (Machado \& Marchiori, 2016a), Mimosa cruenta (Marchiori, 1985; Marchiori et al., 2011), M. uraguensis (Marchiori 1996c; Marchiori et al., 2010) e $M$. trachycarpa (Marchiori \& Muñiz, 1997b).

No tocante a outras séries botânicas, a literatura dispõe de descrições microscópicas dos lenhos de Mimosa bimucronata (DC.) Kuntze (Marchiori, 1993; Marchiori et al., 2010), M. micropteris Benth. (Siegloch et al., 2013), M. parvipinna Benth. (Machado \& Marchiori, 2016b), M. pigra Benth. (Machado \& Marchiori, 2016c), Mimosa ramulosa Benth. (Tomasi et al., 2015; Machado \& Marchiori, 2016b) e Mimosa sparsa Benth. (Maccari \& Marchiori, 1994; Marchiori et al., 2011). Destas, Mimosa parvipinna e Mimosa ramulosa pertencem à subsérie Obstrigosae (Benth.) Barneby (da série Mimosa L. e seção Mimosa L.), Mimosa bimucronata insere-se na série Bimucronatae Barneby (da secão Batocaulon DC.), Mimosa micropterys pertence à série Myriophyllae Benth. (da seção Mimosa L.), Mimosa pigra à série Habbasia DC. (da seção Habbasia DC.), e Mimosa sparsa à subsérie Sparsae Barneby (da série Mimosa L. e seção Mimosa L.). 


\section{MATERIAL E MÉTODOS}

O material examinado consiste de amostras de madeira e exsicatas botânicas, conservadas, respectivamente, na Xiloteca e Herbário do Departamento de Ciências Florestais (HDCF) da Universidade Federal de Santa Maria (RS), e no Herbário Barbosa Rodrigues (HBR - Itajaí, $\mathrm{SC})$, com os seguintes registros:

- HDCF 2890, Mimosa intricata Benth. Leg: Marchiori, J.N.C., 14-09-1986, Caçapava do Sul, RS, à margem da estrada para a Pedra do Segredo.

-- Reitz \& Klein 6.992, Mimosa taimbensis Burkart. Leg.: 21-08-1958, Serra do Oratório, Aparados da Serra, SC, 1400m, flor amarelada.

Do material lenhoso foram preparados três corpos-de-prova $(1 \times 2 \times 3 \mathrm{~cm})$ de cada uma das amostras, orientados para a obtenção de cortes anatômicos nos planos transversal, longitudinal radial e longitudinal tangencial; um quarto bloquinho foi também preparado, com vistas à maceração.

A confeção de lâminas de cortes anatômicos seguiu a metodologia descrita em Burger \& Richter (1991), usando-se, como corantes, uma mistura de Acridina-Vermelha e Crisoidina, num primeiro banho, e Azul-de-Astra no segundo (Dujardin, 1964). No preparo de lâminas de macerado seguiu-se o método de Jeffrey (Freund, 1970), colorindo-se a pasta de fibras apenas com Safranina. Com vistas à montagem permanente, usou-se Entellan nos dois tipos de lâminas.

As descrições basearam-se nas recomendações da IAWA (Wheeler et al., 1989). No caso da percentagem dos tecidos, foram realizadas 600 determinações ao acaso com o auxílio de contador de laboratório, conforme proposto por Marchiori (1980). A abundância de poros foi obtida a partir de um quadrado de área conhecida, superposto, em diferentes pontos, a fotomicrografias tomadas em plano transversal, para ambas as madeiras.

As medições de caracteres anatômicos da madeira foram realizadas em microscópio fotônico Carl Zeiss, no Laboratório de Anato- mia da Madeira da Universidade Federal de Santa Maria. As imagens que ilustram o texto foram tomadas em fotomicroscópio Leica DM 2000, equipado com câmera digital Leica DFC 295, no Laboratório de Botânica Estrutural, do Departamento de Biologia (Centro de Ciências Naturais e Exatas) da Universidade Federal de Santa Maria.

\section{DESCRIÇÃO DAS ESPÉCIES}

Descreve-se, na sequência e por ordem alfabética de nomes científicos, a estrutura microscópica dos lenhos de Mimosa intricata e Mimosa taimbensis.

\section{Mimosa intricata Benth.}

Anéis de crescimento: distintos.

Vasos: muito numerosos (75 \pm 39 (41-200) poros $/ \mathrm{mm}^{2}$ ), ocupando $11 \pm 3,7 \%$ do volume da madeira. Porosidade difusa. Poros racemiformes (53\%), em múltiplos radiais (32\%), menos comumente solitários (15\%); circulares ou ovais $(55 \pm 15(30-85) \mu \mathrm{m})$ e de paredes finas a espessas $(3,5 \pm 1,1(1,2-5) \mu \mathrm{m})$ (Figura 1A,B). Elementos vasculares curtos (128 \pm 34 (70-190) $\mu \mathrm{m})$, com placas de perfuração simples, oblíquas ou transversais ao vaso, e apêndices ausentes, ou então curtos $(29 \pm 13(10-50) \mu \mathrm{m})$. Pontoações intervasculares pequenas e arredondadas $(5,2 \pm 0,3(4,6-5,6) \mu \mathrm{m}$; Figura $1 \mathrm{~F})$. Pontoações raio-vasculares, semelhantes às intervasculares, embora menores $(4,3 \pm 0,4$ (3,6$5,2) \mu \mathrm{m})$. Espessamentos espiralados, ausentes. Conteúdos, presentes em vasos.

Parênquima axial: paratraqueal escasso, representando $10 \pm 6 \%$ do volume da madeira. Conteúdos, não observados (Figura 1A,B). Células parenquimáticas axiais fusiformes e em séries. As fusiformes, de $57 \pm 6,2$ (45-65) $\mu \mathrm{m}$ de altura. Séries parenquimáticas axiais de 212 \pm 53 (138-375) $\mu \mathrm{m}$, com 2-3 células de altura (Figura 1F). Cristais romboédricos em número de 2-22, dispersos em câmaras no parênquima axial.

Raios: muito numerosos $(13 \pm 1,1$ (12-15) raios/mm), ocupando $17 \pm 4 \%$ do volume da 

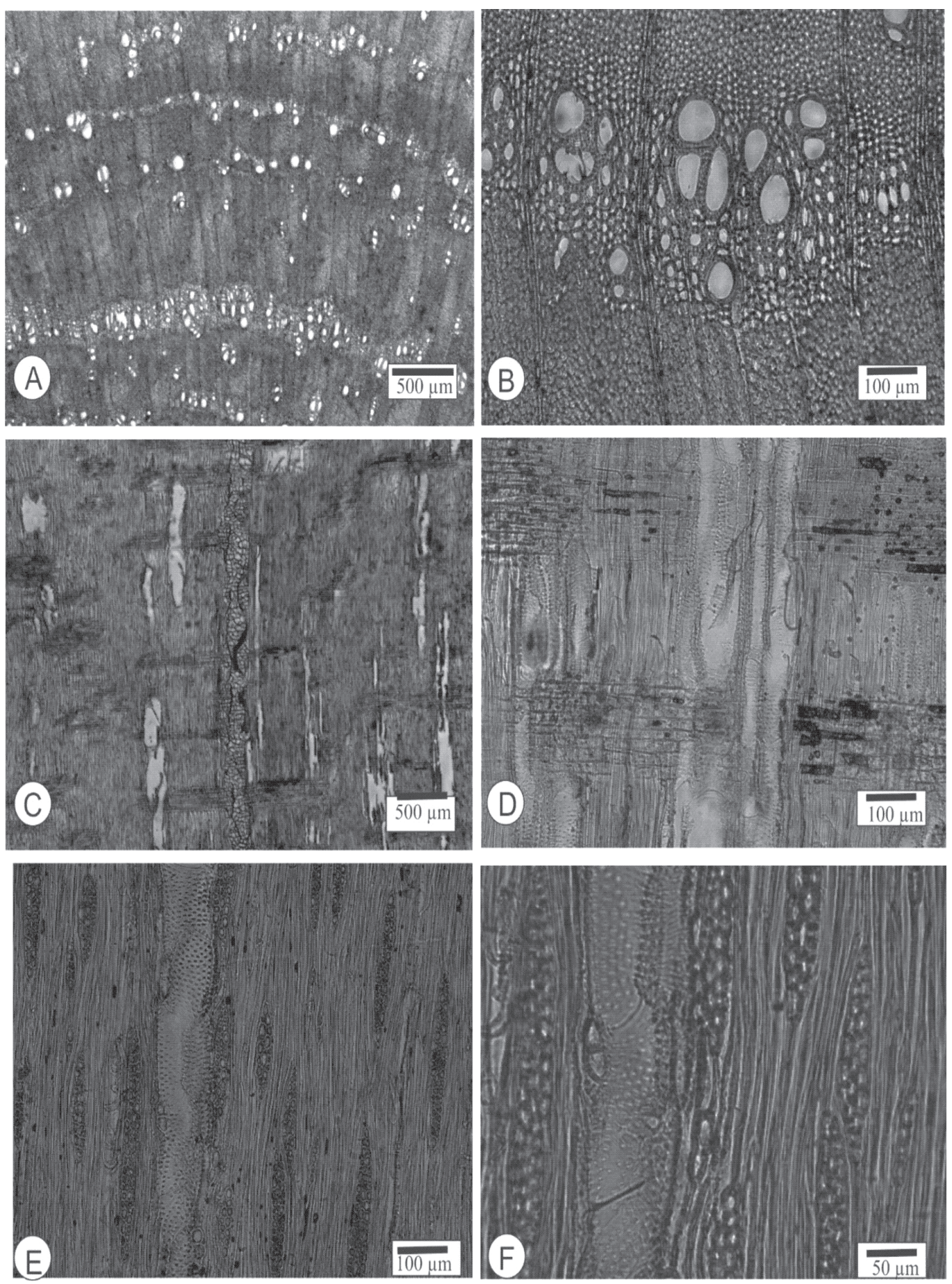

FIGURA 1 - Fotomicrografias do lenho de Mimosa intricata. A - Porosidade difusa, com poros em múltiplos raccemiformes e escassos solitários (seção transversal). B - Poros em múltiplos racemiformes (detalhe) e parênquima paratraqueal escasso (seção transversal). C - Elementos vasculares e raios homogêneos, de células procumbentes (seção radial). D - Raios de células procumbentes e vasos com placas de perfuração simples (seção radial). E - Raios multisseriados, em seção tangencial. F - Placas de perfuração simples, pontoações ornamentadas e raios estreitos, em detalhe (seção tangencial). 
madeira; estreitos $(27 \pm 3(25-35) \mu \mathrm{m})$, homogêneos, compostos inteiramente de células procumbentes (Figura 1C,D). Os unisseriados (14\%), de $55 \pm 24$ (25-125) $\mu \mathrm{m}$, com 2-10 células de altura. Os multisseriados, em sua maioria trisseriados (49\%) e bisseriados (37\%); de 209 \pm 76 (75-350) $\mu \mathrm{m}$, com 7-35 células de altura. Células radiais de contorno arredondado, em plano tangencial. Células envolventes, células radiais de paredes disjuntas e células perfuradas, ausentes. Raios fusionados e conteúdos, presentes.

Fibras: libriformes, de $520 \pm 90$ (300-700) $\mu \mathrm{m}$ de comprimento, com $9 \pm 2(5-12,5) \mu \mathrm{m}$ de largura e paredes finas $(2,9 \pm 0,7(1,9-4,4) \mu \mathrm{m})$, ocupando $62 \pm 7 \%$ do volume da madeira (Figura 1B). Fibras gelatinosas, presentes; espessamentos espiralados, fibras septadas e traqueídeos, ausentes.

Outros caracteres: variantes cambiais, tubos laticíferos e taniníferos, canais intercelulares, células oleíferas, células mucilaginosas e estratificação, ausentes. Máculas medulares, presentes.

\section{Mimosa taimbensis Benth.}

Anéis de crescimento: distintos.

Vasos: muito numerosos (99 \pm 33 (57-167) poros $/ \mathrm{mm}^{2}$ ), ocupando $17 \pm 3 \%$ do volume da madeira. Porosidade difusa. Poros, principalmente em múltiplos radiais (52\%) e solitários (27\%), com escassos racemiformes de poucas unidades $(21 \%)$; circulares ou ovais $(58 \pm 8,5$ $(38-68) \mu \mathrm{m})$ e de paredes finas a espessas $(3,4$ $\pm 0,9(1,2-5) \mu \mathrm{m})$ (Figura 2A,B). Elementos vasculares curtos $(200 \pm 58(110-390) \mu \mathrm{m})$, geralmente esprovidos de apêndices e com placas de perfuração simples, oblíquas ou transversais ao vaso. Pontoações intervasculares pequenas e arredondadas $(5,6 \pm 0,5(5,2-6,2) \mu \mathrm{m})$ (Figura $2 \mathrm{~F})$. Pontoações raio-vasculares, semelhantes às intervasculares, embora menores $(3,8 \pm 0,7$ (3$5,2) \mu \mathrm{m})$. Espessamentos espiralados, ausentes. Conteúdos, presentes.

Parênquima axial: paratraqueal escasso, representando $13 \pm 5,7 \%$ do volume da madeira.
Conteúdos, não observados (Figura 2A,B). Células parenquimáticas fusiformes e em séries axiais. As fusiformes, de $207 \pm 38$ (125-275) $\mu \mathrm{m}$ de altura. Séries parenquimáticas axiais de $280 \pm 99(163-463) \mu \mathrm{m}$ de altura, com $3 \pm 1$ (25) células (Figura 2F). Cristais romboédricos em número de 2-20, dispostos em câmaras individuais.

Raios: numerosos (7 \pm 1 (6-9) raios $/ \mathrm{mm}$ ), ocupando $16 \pm 4,4 \%$ do volume da madeira; largos (56 \pm 12 (25-73) $\mu \mathrm{m})$ e homogêneos, compostos, inteiramente, de células procumbentes (Figura 2C,D). Os unisseriados (11\%), de $14 \pm$ $2(13-18) \mu \mathrm{m}$, com 2-12 células de altura. Os multisseriados, em sua maioria tetrasseriados $(53 \%)$ e trisseriados $(20 \%)$, menos comumente bisseriados $(7 \%)$ e com mais de 5 células de largura (9\%); de $690 \pm 317$ (250-1443) $\mu \mathrm{m}$ de altura, com 13-76 células. Células radiais de contorno arredondado, em plano tangencial. Células envolventes, células radiais de paredes disjuntas e células perfuradas, ausentes. Raios fusionados e conteúdos, presentes.

Fibras: libriformes, de $595 \pm 78$ (440-730) $\mu \mathrm{m}$ de comprimento, com $12 \pm 2,2(7,5-16) \mu \mathrm{m}$ de largura e paredes finas $(2,9 \pm 0,5(2,5-4,4)$ $\mu \mathrm{m})$, ocupando $54 \pm 6,2 \%$ do volume da madeira (Figura 2B). Fibras gelatinosas, presentes. Fibras septadas, espessamentos espiralados e traqueídeos, ausentes.

Outros caracteres: variantes cambiais, tubos laticíferos e taniníferos, canais intercelulares, células oleíferas e células mucilaginosas, ausentes; máculas medulares, não observadas. Estratificação parcial, de parênquima axial e elementos vasculares, em plano tangencial.

\section{ANÁLISE ANATÔMICA}

Tanto no lenho de Mimosa intrincata como no de Mimosa taimbensis se observa o conjunto de caracteres anatômicos reconhecido por Record \& Hess (1949) como típico de Leguminosae (Fabaceae): é o caso, entre outros, da ocorrência de placas de perfuração simples, de pontoações intervasculares ornamentadas e em arranjo alterno, de parênquima paratraqueal 

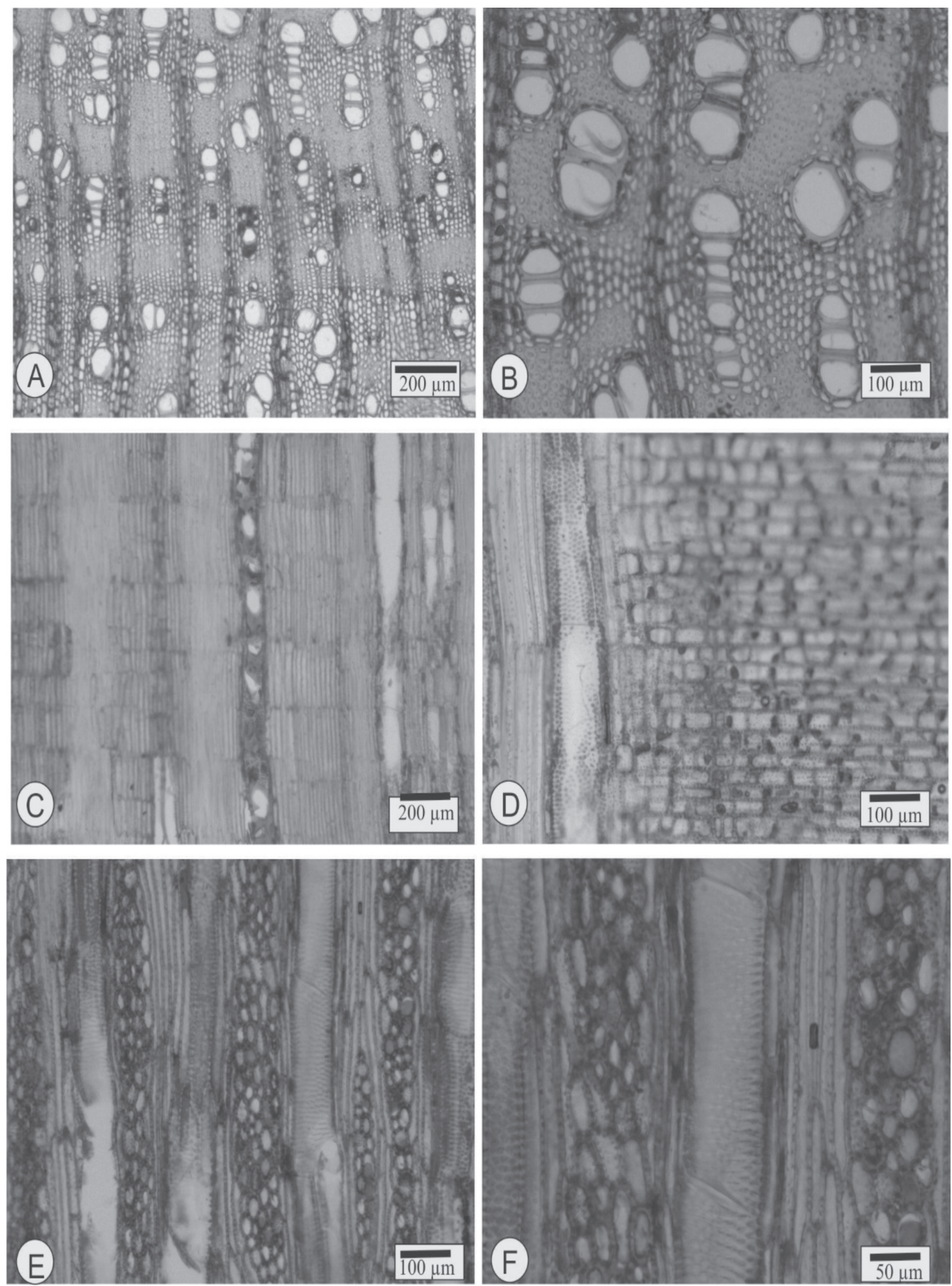

FIGURA 2 - Fotomicrografias do lenho de Mimosa taimbensis. A - Porosidade difusa, com poros solitários e escassos múltiplos radiais (seção transversal). B - Poros em múltiplos radiais, em detalhe (seção transversal). C - Raios homogêneos, de células procumbentes, elementos vasculares e parênquima axial seriado (seção radial). D - Raios de células procumbentes e placas de perfuração simples, em plano radial. E - Raios multisseriados e vasos, em seção tangencial. F - Placas de perfuração simples, pontoações ornamentadas em arranjo alterno e raios largos (seção tangencial). 
e de fibras libriformes. Por sua vez, a presença de raios homogêneos e de células procumbentes, anotado para ambas as espécies e que se mostra predominante em Mimosaceae, segundo Metcalfe \& Chalk (1972), atesta, de acordo com Baretta-Kuipers (1981), elevada especialização aos raios de Mimosoideae.

Sobre esse último ponto, todavia, cabe salientar que em algumas espécies do gênero se observam células procumbentes e quadradas (raios heterogêneos ou fracamente heterogêneos), como em Mimosa daleoides (Marchiori, 1982), Mimosa ramulosa (Tomasi et al., 2015; Machado \& Marchiori, 2016b) e Mimosa parvipinna (Machado \& Marchiori, 2016b). Foi pela diversidade estrutural relativamente alta do lenho, aliás, que Cozzo (1951) equiparou a anatomia de Mimosa com a do conjunto das Mimosoideae. Esta diversidade estrutural, por sua vez, justifica, sob o ponto de vista da anatomia da madeira, a recente incorporação das Mimosoideae na subfamília Caesalpinioideae, recomendada pelo LPWG (2017), com base em genética molecular.

O arranjo de vasos também ilustra a diversidade estrutural apontada, por Cozzo (1951), para o gênero Mimosa. Neste estudo, a predominância de poros em múltiplos racemiformes se mostra suficiente para separar Mimosa intricata de Mimosa taimbensis.

A predominância de múltiplos racemiformes em Mimosa intricata assume valor diagnóstico para o reconhecimento anatômico da série Stipellares Benth., uma vez que o mesmo se restringe, aparentemente, a espécies desse grupo, salientando-se Mimosa barnebiana e M. bifurca (Machado \& Marchiori, 2016a), Mimosa cruenta (Marchiori, 1985; Marchiori et al, 2011), Mimosa trachycarpa (Marchiori \& Muñiz, 1997b), Mimosa uraguensis (Marchiori, 1966c; Marchiori et al., 2010) e Mimosa uliginosa (Cozzo, 1951). Resta acrescentar que o último autor também anotou o caráter para Mimosa bifurca (sob o nome de Mimosa hassleriana) e Mimosa cruenta.
A ocorrência de poros solitários e em múltiplos de poucas unidades, presentemente observada em Mimosa taimbensis, é caráter predominante nos lenhos de Mimosa L., ocorrendo tanto em espécies da seção Calothamnos Barneby, caso de Mimosa scabrella (Marchiori, 1995; Marchiori et al., 2009; Richter \& Charvet, 1973; Tortorelli, 1956), como das seções Batocaulon DC. (Mimosa bimucronata, por exemplo; ver: Marchiori, 1993; Marchiori et al., 2010), Habbasia DC. (Mimosa pigra, por exemplo; ver: Machado \& Marchiori, 2016c), e Mimosa L. (Mimosa sparsa, por exemplo; ver: Maccari \& Marchiori, 1994).

A estratificação de parênquima axial e elementos vasculares em Mimosa taimbensis é caráter pouco frequente no gênero. Registrado, por Cozzo (1949), para M. scabrella (seção Calothamnos Barneby) e M. adpressa (subsérie Obstrigosae (Benth.) Barneby, série Mimosa L., seção Mimosa L.), o mesmo é amplamente reconhecido como indicador de elevada especialização no xilema secundário.

\section{REFERÊNCIAS BIBLIOGRÁFICAS}

BARETTA-KUIPERS, T. Wood anatomy of Leguminosae: its relevance to Taxonomy. In: POLHILL, R.M.; RAVEN, P.H. Advances in Legume Systematics. Kew: Royal Botanic Gardens, 1981. p. 677-715.

BARNEBY, R.C. Sensitivae Censitae. A description of the genus Mimosa Linnaeus (Mimosaceae) in the New World. Memoirs of the New York Botanical Garden, v. 65, p. 1-835, 1991.

BENTHAM, G. Notes on Mimoseae, with a synopsis of species. The London Journal of Botany, n. 1, p.318-392; 494-518, 1842.

BENTHAM, G. Leguminosae. III. Mimoseae. In: MARTIUS, CF.O. von. Flora Brasiliensis, v. 15, n. 2 , p. $259-527,1876$.

BESSEGA, C.; HOPP, H.E.; FORTUNATO, R.H. Toward a phylogeny of Mimosa (Leguminosae: Mimosoideae): a preliminary analysis of southern South American species based on chloroplast DNA sequence. Annals of the Missouri Botanical Garden, St. Louis, n. 95, p. 567-579, 2008. 
BURGER, L.M.; RICHTER, H.G. Anatomia da Madeira. São Paulo: Ed. Nobel, 1991. 154 p.

BURKART, A. Las Leguminosas argentinas silvestres y cultivadas. Buenos Aires: ACME, 1952. 569 p.

BURKART, A. Sinopsis de las especies de Mimosa de la serie Lepidotae. Darwiniana, San Isidro, v. 13, n. 2-4, p. 362-365, 1964.

CARNIELETTO, C.; MARCHIORI, J.N.C. Anatomia da madeira de Mimosa eriocarpa Benth. Ciência Florestal, Santa Maria, v. 3, n. 1, p. 107-120, 1993.

COUTINHO, A.P.S. Revisão taxonômica e estudos filogenéticos de Mimosa L. sect. Calothamnos Barneby (Leguminosae-Mimosoideae). São Paulo: Universidade de São Paulo, 2009. Doutorado em Botânica, Tese. 311 p.

COZZO, D. Anatomia del leño secundario de las Leguminosas Mimosoideas y Caesalpinoideas argentinas silvestres y cultivadas. Rev. Inst. Nac. Invest. Ci. Nat. Ci. Bot., Buenos Aires, v. 2, n. 2, p. 63-290, 1951.

COZZO, D. Estructura leñosa estratificada no registrada en generos de Leguminosas argentinas. Lilloa, Tucumán, v. 16, p. 63-95, 1949.

DUJARDIN, E.P. Eine neue HolzZellulosenfaerbung. Mikrokosmos, n. 53, p. 94, 1964.

FREUND, H. Handbuch der Mikroskopie in der Technik. Frankfurt: Umsham Verlag, 1970. 375p.

IZAGUIRRE, P.; BEYHAUT, R. Las Leguminosas en Uruguay y regiones vecinas. Montevideo: Editorial Hemisfério Sur, 2003. 301 p.

KLEIN, R.M. Observações ecológicas. In: BURKART, A. Leguminosas Mimosoideas. Itajaí: Herbário Barbosa Rodrigues, 1979. p. 167-168.

LPWG - The Legume PhylogenyWorking Group. A new subfamily classification of the Leguminosae based on a taxonomically comprehensive phylogeny. Taxon, v. 66, n. 1, p. 44-77, 2017.

LUCKOW, M.A.; WHITE, P.W.; BRUNEAU, A. Relationships among the basal genera of Mimosoid Legumes. In: HERENDEEN, P.S.; BRUNEAU, S. (eds.). Advances in Legume Systematics. Kew: Royal Botanic Gardens, 2000, v. 9, p. 165-180.

MACCARI, A.; MARCHIORI, J.N.C. Estudo anatômico do xilema secundário de Mimosa sparsa Benth. Ciência Florestal, Santa Maria, v. 4, n. 1, p. 145-155, 1994.

MACHADO, P.F. dos S.; MARCHIORI, J.N.C. Anatomia da madeira de duas espécies de Mimosa da série Stipellares Benth. Balduinia, Santa Maria, n. 54, p. 1-10, 2016 a.

MACHADO, P.F. dos S.; MARCHIORI, J.N.C. Anatomia do lenho de duas espécies sulbrasileiras do gênero Mimosa L e subsérie Obstrigosae (Benth.) Barneby. Balduinia, Santa Maria, n. 52, p. 22-29, 2016 b.

MACHADO, P.F. dos S.; MARCHIORI, J.N.C. Anatomia do lenho de Mimosa pigra. Balduinia, Santa Maria, n. 55, p. 17-22, $2016 \mathrm{c}$.

MARCHIORI, J.N.C. Estudo anatômico do xilema secundário e da casca de algumas espécies dos gêneros Acacia e Mimosa, nativas no estado do Rio Grande do Sul. Dissertação (Mestrado em Engenharia Florestal). Curitiba: Universidade Federal do Paraná, 1980. 186 f.

MARCHIORI, J.N.C. A estrutura do xilema secundário de Mimosa daleoides Benth. (Leguminosae Mimosoideae). Ciência e Natura, Santa Maria, n. 4, p. 107-113, 1982.

MARCHIORI, J.N.C. Anatomia da madeira de Mimosa cruenta Benth. (Leguminosae Mimosoideae). Ciência e Natura, Santa Maria, n. 7, p. 73-81, 1985.

MARCHIORI, J.N.C. Anatomia da madeira e casca do maricá, Mimosa bimucronata (DC.) Kuntze. Ciência Florestal, Santa Maria, v. 3, n. 1, p. 85106, 1993.

MARCHIORI, J.N.C. Anatomia da madeira e casca da bracatinga, Mimosa scabrella Benth. Ciência e Natura, Santa Maria, n. 17, p. 115-132, 1995.

MARCHIORI, J.N.C. Anatomia do xilema secundário de Mimosa berroi Burk. Ciência e Natura, Santa Maria, n. 18, p. 117-129, 1996 a.

MARCHIORI, J.N.C. Anatomia do xilema secundário de Mimosa incana (Spreng.) Benth. Ciência Florestal, Santa Maria, v. 6, n. 1, p. 5363, $1996 \mathrm{~b}$.

MARCHIORI, J.N.C. Anatomia do xilema secundário de Mimosa uruguensis Hook. \& Arn. Ciência e Natura, Santa Maria, n. 18, p. 103115, 1996c.

MARCHIORI, J.N.C.; MUÑIZ, G.I.B. de. Estudo anatômico do xilema secundário de Mimosa pilulifera Benth. Ciência Florestal, Santa Maria, v. 7, n. 1, p. 65-75, 1997a. 
MARCHIORI, J.N.C.; MUÑIZ, G.I.B. de. Estudo anatômico do xilema secundário de Mimosa trachycarpa Benth. Ciência Rural, Santa Maria, v. 27, n. 2, p. 223-228, 1997b.

MARCHIORI, J.N.C.; MUÑIZ, G.I.B. de; SANTOS, S.R. dos. Madeiras do Rio Grande do Sul. 1 Descrição microscópica de 33 espécies nativas. Santa Maria: [s.n.], 2009. 80 p.

MARCHIORI, J.N.C.; MUÑIZ, G.I.B. de; SANTOS, S.R. dos. Madeiras do Rio Grande do Sul. 2 Descrição microscópica de 35 espécies nativas. Santa Maria: Anaterra, 2010. 80 p.

MARCHIORI, J.N.C.; DENARDI, L.; SANTOS, S.R. dos. Madeiras do Rio Grande do Sul. 3 Descrição microscópica de 34 espécies nativas. Santa Maria: Anaterra, 2011. 80 p.

METCALFE, C.R.; CHALK. L. Anatomy of the Dicotyledons. Oxford: Clarendon Press, 1972. $1500 \mathrm{p}$.

PEREIRA, A.C. de S.; SIEGLOCH, A.M.; MARCHIORI, J.N.C. Anatomia do lenho de Mimosa flocculosa Burkart. Balduinia, Santa Maria, n. 32, p. 29-33, 2013.

RAMBO, B. Leguminosae riograndenses. Pesquisas, Botânica, n. 23, p. 33, 1966.

RECORD, S.J.; HESS, R.W. Timbers of the New World. New Haven: Yale University Press, 1949. 640 p.

REINDERS-GOUWENTAK, C.A. The storied structure features and the taxonomic rank of the Leguminous taxa. Acta Botanica Neerlandica, n. 4, p. 460-470, 1955.

RICHTER, H.G.; CHARVET, L.M. Estudo e pesquisa sobre Mimosa scabrella. Floresta, Curitiba, v. 4, n. 2, p. 68-69, 1973.

SANTOS-SILVA, J.; FRAGOMENI, S.M.; TOZZI, A.M.G. de A. Revisão taxonômica das espécies de Mimosa ser. Leiocarpae sensu lato
(Leguminosae - Mimosoideae). Rodriguésia, Rio de Janeiro, v. 66, n. 1, p. 95-154, 2015.

SENN, H.A. The relation of Anatomy and Cytology to the classification of the Leguminosae. Chron. Bot., n. 7, p. 306-308, 1943.

SIEGLOCH, A.M.; MARCHIORI, J.N.C.; SANTOS, S.R. dos. Anatomia do lenho de Mimosa micropteris Benth. Balduinia, Santa Maria, n. 40, p. 18-22, 2013.

SIMON, M.F. Systematics and evolution of Mimosa L. (Leguminosae) and the assembly of a neotropical plant diversity hotspots. Oxford: Oxford University, 2008 (Tese de Doutorado).

SIMON, M.F.; GRETHER, R.; QUEIROZ, L.P.; SÄRKINEN, T.E.; DUTRA, V.F.; HUGHES, C.E. The evolutionary history of Mimosa (Leguminosae): toward a phylogeny of the sensitive plants. American Journal of Botany, St. Louis, v. 98, n. 7, p. 1201-1221, 2011.

SULAIMAN, S.F.; CULHAM, A.; HARBORNE, J.B. Molecular Phylogeny of Fabaceae based on rbcL sequence data: With special emphasis on the tribe Mimoseae (Mimosoideae). Asia Pacific Journal of Molecular Biology and Biotehnology, Kuala Lampur, v. 11, n. 1, p. 9-35, 2003.

TOMASI, J. de C.; DENARDI, L.; TREVISAN, R.; TRAMONTINA, J.; BALDIN, T. Descrição anatômica do xilema secundário de Mimosa ramulosa Benth. (Fabaceae). Enciclopédia Biosfera, Centro Científico Conhecer, Goiânia, v. 112, n. 21, p. 727-736, 2015.

TORTORELLI, L.A. Maderas y bosques argentinos. Buenos Aires: ACME, 1956. 910 p.

WHEELER, E.A.; BAAS, P.; GASSON, P.E. IAWA list of microscopic features for hardwood identification. IAWA Bulletin, v. 10, n. 3, p. 218$359,1989$. 